11-2014

\title{
Survival Estimation Using Bootstrap, Jackknife and K-Repeated Jackknife Methods
}

Johnson A. Adewara

University of Lagos, Akoka-Lagos, Nigeria, jadewara@unilag.edu.ng

Ugochukwu A. Mbata

University of Lagos, Akoka-Lagos, Nigeria, mmbataugochukwu@yahoo.com

Follow this and additional works at: http://digitalcommons.wayne.edu/jmasm

Part of the Applied Statistics Commons, Social and Behavioral Sciences Commons, and the Statistical Theory Commons

\section{Recommended Citation}

Adewara, Johnson A. and Mbata, Ugochukwu A. (2014) "Survival Estimation Using Bootstrap, Jackknife and K-Repeated Jackknife Methods," Journal of Modern Applied Statistical Methods: Vol. 13 : Iss. 2 , Article 15.

DOI: $10.22237 /$ jmasm/1414815240

Available at: http://digitalcommons.wayne.edu/jmasm/vol13/iss2/15

This Regular Article is brought to you for free and open access by the Open Access Journals at DigitalCommons@WayneState. It has been accepted for inclusion in Journal of Modern Applied Statistical Methods by an authorized editor of DigitalCommons@WayneState. 


\section{Survival Estimation Using Bootstrap, Jackknife and K-Repeated Jackknife Methods}

\author{
Johnson Ademola Adewara \\ University of Lagos \\ Akoka-Lagos, Nigeria
}

\author{
Ugochukwu Ahamefula Mbata \\ University of Lagos \\ Akoka-Lagos, Nigeria
}

Three re-sampling techniques are used to estimate the survival probabilities from an exponential life-time distribution. The aim is to employ a technique to obtain a parameter estimate for a two-parameter exponential distribution. The re-sampling methods considered are: Bootstrap estimation method (BE), Jackknife estimation method (JE) and the $k$-repeated Jackknife estimation method (KJE). The methods were computed to obtain the mean square error (MSE) and mean percentage error (MPE) based on simulated data. The estimates of the two-parameter exponential distribution were substituted to estimate survival probabilities. Results show that the MSE value is reduced when the $K$-repeated jackknife method is used.

\section{Introduction}

Modern statistics is anchored in the use of statistics and hypothesis tests that only have desirable and well-known properties when computed from populations that are normally distributed. While it is claimed that many such statistics and hypothesis tests are generally robust with respect to non-normality, other approaches that require an empirical investigation of the underlying population distribution or of the distribution of the statistic are possible and in some instances preferable. In instances when the distribution of a statistic, conceivably a very complicated statistic, is unknown, no recourse to a normal theory approach is available and alternative approaches are required. Statistical models and methods for estimating survival data and other time-to-event data are extensively used in many fields, including the biomedical sciences, engineering, the environmental sciences, economics, actuarial sciences, management, and the social sciences.

Johnson Ademola Adewara is a Senior Lecturer at the Distance Learning Institute at the University of Lagos. Email at jadewara@unilag.edu.ng

U.A. Mbata is a Graduate Fellow and Consulting Statistician in the Mathematics

Department at the University of Lagos. Email at mmbataugochukwu@yahoo.com 


\section{SURVIVAL ESTIMATION USING THREE METHODS}

Survival analysis refers to techniques for studying the occurrence and timing of events. It is concerned with studying the random variable $T$, representing the time between entry to a study and some event of interest, such as: death, the onset of disease, time until equipment failures, earthquakes, automobile accidents, time-topromotions, time until stock market crashes, revolutions, job terminations, births, marriages, divorces, retirements or arrests. There are many different models for survival data, and what often distinguishes one model from another is the probability distribution for $T$. Resampling statistics refer to the use of the observed data or of a data generating mechanism (such as a die) to produce new hypothetical samples (resamples) that mimic an underlying population, the results of which can then be analyzed. With numerous cross-disciplinary applications especially in the sub-disciplines of the life science, resampling methods are widely used because they are options when parametric approaches are difficult to employ or otherwise do not apply.

Resampled data is derived using a manual mechanism to simulate many pseudo-trials. These approaches were difficult to utilize prior to $1980 \mathrm{~s}$ because these methods require many repetitions. With the incorporation of computers, the trials can be simulated in a few minutes and is why these methods have become widely used. The methods that will be discussed are used to make many statistical inferences about the underlying population. The most practical use of resampling methods is to derive confidence intervals and test hypotheses. This is accomplished by drawing simulated samples from the data themselves (resamples) or from a reference distribution based on the data; afterwards, you are able to observe how the statistic of interest in these resamples behaves. Resampling approaches can be used to substitute for traditional statistical (formulaic) approaches or when a traditional approach is difficult to apply. These methods are widely used because their ease of use. They generally require minimal mathematical formulas, needing a small amount of mathematical (algebraic) knowledge. These methods are easy to understand and stray away from choosing an incorrect formula in your diagnostics.

Two general approaches considered here are: Jackknife approach and Bootstrap approach. The aim of this study is to employ a technique to obtain an estimate of the parameter of the two-parameter exponential distribution. The methods considered in this paper are: Bootstrap estimation method (BE), Jackknife estimation method (JE) and the $k$-repeated Jackknife estimation method (KJE). The estimates of the two-parameter exponential distributions are used to estimate the survival probability. Methodology under Bootstrap, Jackknife and the proposed Krepeated Jackknife is presented, followed by data analysis, results, discussion and a conclusion. 


\section{Two-Parameter Exponential Distribution}

Re-sampling methods are becoming increasingly popular as statistical tools. These methods involve sampling or scrambling the original data numerous times. Two general approaches are considered here. They are: Jackknife approach and Bootstrap approach. The two-parameter exponential distribution is adopted when failure will never occur prior to some specified time, $t_{0}$. The parameter $t_{0}$ is a location parameter that shifts the distribution an amount equal to $t_{0}$ towards the right on the time line. When $t \geq t_{0}$, the probability density function of exponential distribution becomes:

$$
f(t ; \theta)=\frac{1}{\theta} \exp \left(-\frac{1}{\theta}\left(t-t_{0}\right)\right), t \geq 0, \theta>0, t \geq t_{0}
$$

and the survival function is given by:

$$
S(t)=\int_{t}^{\infty} f(t ; \theta) d t
$$

where $\lambda=\frac{1}{\theta}$.

\section{Bootstrap Estimation Method}

Bootstrapping is a modern, computer-intensive, general purpose approach to statistical inference, falling within a broader class of re-sampling methods to simplify the often intricate calculations of traditional statistical theory. A parametric bootstrap method is considered in this article.

The general theory (see Rizzo, 2008) is as follows. Suppose $t_{1}, t_{2}, \ldots, t_{n}$ is a random sample from the distribution of $T$. An estimator $\hat{\theta}$ for a parameter $\theta$ is an $\mathrm{n}$ variate function $\hat{\theta}=\hat{\theta}\left(t_{1}, t_{2}, \ldots, t_{n}\right)$ of the sample. Functions of the estimator $\hat{\theta}$ are therefore $\mathrm{n}$-variate functions of the data, also. For simplicity, let $t=\left(t_{1}, t_{2}, \ldots, t_{n}\right)^{T} \in R^{n}$, and $t^{(1)}, t^{(2)}, \ldots$ denote a sequence of independent random samples generated from the distribution of $T$. Random variables from the sampling distribution of $\hat{\theta}$ can be generated by repeatedly drawing independent random samples $t^{(j)}$ and computing $\hat{\theta}^{(j)}=\hat{\theta}\left(t_{1}^{(j)}, t_{2}^{(j)}, \ldots, t_{n}^{(j)}\right)$ for each sample. The mean of the replicates is given as 


\section{SURVIVAL ESTIMATION USING THREE METHODS}

$$
\hat{\theta}_{B}=\frac{1}{m} \sum_{i=1}^{m} \hat{\theta}^{(j)}
$$

The mean squared error (MSE) is defined by $\operatorname{MSE}(\hat{\theta})=E\left[(\hat{\theta}-\theta)^{2}\right]$. If $\mathrm{m}$ random samples $t^{(1)}, t^{(2)}, \ldots, t^{(m)}$ are generated from the distribution of $T$ then estimate of the MSE of $\hat{\theta}=\hat{\theta}\left(t_{1}, t_{2}, \ldots, t_{n}\right)$ is

$$
\operatorname{MSE}\left(\hat{\theta}_{B}\right)=\frac{1}{m-1} \sum_{j=1}^{m}\left(\hat{\theta}^{(j)}-\theta\right)^{2}
$$

where $\hat{\theta}^{(j)}=\hat{\theta}\left(t_{1}^{(j)}, t_{2}^{(j)}, \ldots, t_{n}^{(j)}\right)$.

Estimate of the standard error of the bootstrap estimate, $\hat{\theta}_{B}$ is given by

$$
S E\left(\hat{\theta}_{B}\right)=\sqrt{\frac{1}{m-1} \sum_{j=1}^{m}\left(\hat{\theta}^{(j)}-\theta\right)^{2}}
$$

$100(1-\alpha) \%$ confidence interval for $\theta$ is given by

$$
\hat{\theta}_{B} \pm Z_{\alpha / 2} S E\left(\hat{\theta}_{B}\right)
$$

The mean percentage error (MPE) is

$$
\operatorname{MPE}\left(\hat{\theta}_{B}\right)=\frac{\left[\sum_{j=1}^{m} \frac{\left|\hat{\theta}^{(j)}-\theta\right|}{\theta}\right]}{m} .
$$

If the bootstrap estimator $\hat{\theta}_{B}$ is known from (3) then the estimate of survival function is given as

$$
\hat{S}_{B}(t)=\exp \left(-\frac{t_{i}-t_{0}}{\hat{\theta}_{B}}\right)
$$




\section{ADEWARA \& MBATA}

\section{Jackknife Estimation}

The jackknife is a more orderly version of the bootstrap. As opposed to re-sampling randomly from the entire sample like the bootstrap does, the jackknife takes the entire sample except for 1 value, and then calculates the test statistic of interest. It repeats the process, each time leaving out a different value, and each time recalculating the test statistic. This method was introduced by Quenouille (1949) and further modification in Quenouille (1956). The theory is as follows:

Let $\hat{\theta}$ be an estimator of the parameter $\theta$ based on the complete sample of size $\mathrm{n}$ with $g$ subgroups. Let $\hat{\theta}_{-i}$ be the corresponding estimator based on the sample at the $i^{\text {th }}$ deletion. Define

$$
\tilde{\theta}_{i}=g \hat{\theta}-(g-1) \hat{\theta}_{-i}(i=1,2, \ldots, g)
$$

The $i^{\text {th }}$ deletion of the total could be one individual observation or several observation. The latter case is called group- or block-based jackknife if one replication or one block observations are deleted. In equation (1) estimation $\tilde{\theta}_{i}$ is called the $i^{\text {th }}$ pseudo value and the estimator in equation (2) is the jackknife estimator for the parameter $\theta$, where $\theta$ can be a variance component, covariance component, correlation coefficient, or any other parameter of interest.

$$
\tilde{\theta}=\frac{1}{g} \sum_{j=1}^{g} \tilde{\theta}_{i}=g \hat{\theta}-(g-1) \frac{1}{g} \sum_{j=1}^{g} \hat{\theta}_{-i}
$$

In equation (10), $\tilde{\theta}$ is called a pseudo jackknife estimate. A t-test can then be used to test significant deviation from a given parameter value, $\theta_{0}$ with degrees of freedom $g-1$ (Miller, 1974a, b). The equation (9) can be rewritten as

$$
\tilde{\theta}_{i}=g \hat{\theta}-(g-1) \hat{\theta}_{-i}=\hat{\theta}+(g-1)\left(\hat{\theta}-\hat{\theta}_{-i}\right)(i=1,2, \ldots, g)
$$

Thus, it is obvious that pseudo value $\tilde{\theta}_{i}$ in equation (11) is related to choices for $g$. When $g$ is large, a slight difference between $\hat{\theta}$ and $\hat{\theta}_{-i}$ will cause unfavorable values. More importantly, it will potentially cause a large standard error for an estimate and thus decrease the power for the parameter being tested. If it is assumed that the estimate $\hat{\theta}_{-i}$ in equation (9) for the $i^{\text {th }}$ deletion is unbiased, 


\section{SURVIVAL ESTIMATION USING THREE METHODS}

then it is easy to prove that $\overline{\hat{\theta}}$ in equation (12) is unbiased too. It is often true if $\hat{\theta}$ is an unbiased estimate of $\theta$, then $\hat{\theta}_{-i}$ will be unbiased after a few individuals in the original data are deleted.

$$
\overline{\hat{\theta}}_{\text {jack }}=\frac{\sum_{i=1}^{g} \hat{\theta}_{-i}}{g} .
$$

In equation (12), $\overline{\hat{\theta}}$ is called a non-pseudo jackknife estimate of the parameter $\theta$. For each non-normally distributed variable, based on the Central Limit Theorem, $\overline{\hat{\theta}}$ is approximately normally distributed when $g$ is large. Thus, an approximate $\mathrm{z}-$ test can be used when $g$ is large or t-test can be used to test significant deviation from a given parameter value, $\theta_{0}$, with the degrees of freedom $g-1$. An estimate of the mean square error (MSE) of the jackknife estimate, $\overline{\hat{\theta}}_{\text {jack }}$ is given by

$$
\operatorname{MSE}\left(\overline{\hat{\theta}}_{j a c k}\right)=\frac{g}{g-1} \sum_{i=1}^{g}\left(\hat{\theta}_{-i}-\overline{\hat{\theta}}\right)^{2}
$$

Estimate of the standard error of the jackknife estimate, $\overline{\hat{\theta}}_{\text {jack }}$ is given by

$$
S E\left(\overline{\hat{\theta}}_{j a c k}\right)=\sqrt{\frac{g}{g-1} \sum_{i=1}^{g}\left(\hat{\theta}_{-i}-\overline{\hat{\theta}}\right)^{2}}
$$

$100(1-\alpha) \%$ confidence interval for $\theta$ is given by

$$
\overline{\hat{\theta}}_{j a c k} \pm t_{\alpha / 2, g-1} S E\left(\overline{\hat{\theta}}_{j a c k}\right)
$$

The mean percentage error (MPE) is

$$
\operatorname{MPE}\left(\hat{\theta}_{j a c k}\right)=\frac{\left[\sum_{i=1}^{g} \frac{\left|\hat{\theta}_{-i}-\overline{\hat{\theta}}\right|}{\overline{\hat{\theta}}}\right]}{g} .
$$


If the jackknife estimator $\hat{\theta}_{\text {jack }}$ is known from (12) then the estimate of survival function is given as

$$
\hat{S}_{\text {jack }}(t)=\exp \left(-\frac{t_{i}-t_{0}}{\overline{\hat{\theta}}_{\text {jack }}}\right)
$$

\section{K- Repeated Jackknife Estimation Method}

The $K$ - Repeated jackknife procedure is a re-sampling iterative scheme for mean square error (MSE) reduction. This involves jackknifing the observed data $k$-time, where $k$ equals the sample size of the observed data. The procedure is conveniently applied when the sample size is small. The stopping rule for the repeated jackknife replications depends on the sample size of the original data. The procedure converges before or at $k^{\text {th }}$ time, where the estimate from the jackknife replications is the same as estimator of the parameter $\theta$ based on the complete sample of size $n$. At the $K^{\text {th }}$ time, the $k^{\text {th }}$ - repeated jackknife estimate of bias is highly negligible.

The method involves the following steps from the usual jackknife procedure:

Step 1. Observe a random sample $T=\left(t_{1}, t_{2}, \ldots, t_{n}\right)$

Step 2. Compute $\hat{\theta}(t)$ a function of the data which estimates the parameter $\theta$ of the model.

$$
\hat{\theta}=\frac{1}{n} \sum_{i=1}^{n} t_{i} \quad i=1,2, \ldots, n
$$

Step 3. For $i$ up to $n$

- $\quad$ generate a jackknife sample $T_{-i}=\left(t_{1}, t_{i-1}, t_{i+1}, \ldots, t_{n}\right)$ by leaving out the $i^{\text {th }}$ observation

- $\quad$ calculate $\hat{\theta}_{-i}$ from each of the Jackknife sample $T_{-i}$ by

$$
\hat{\theta}_{-i}=\frac{1}{n-1} \sum_{i=1}^{n-1} T_{-i}
$$




\section{SURVIVAL ESTIMATION USING THREE METHODS}

Step 4. Repeat step 3 using the estimates from $\hat{\theta}_{-i}$ to form pseudo samples. The new pseudo samples are used to generate another set of jackknife estimates; this is continued until the $k^{\text {th }}$ time. This implies that the process is repeated $k$ times, and at any given stage the preceding jackknife estimates are used as new samples in the next stage until the $k^{\text {th }}$ time.

Step 5. At the $k^{\text {th }}$ time the $K$-repeated Jackknife estimate is calculated as

$$
\overline{\hat{\theta}}^{K}=\frac{1}{k} \sum_{i=1}^{n} \hat{\theta}_{i-1}^{K}
$$

The $K$ - repeated jackknife estimate of mean square error (MSE) is given by

$$
\operatorname{MSE}\left(\overline{\hat{\theta}}^{K}\right)=\frac{1}{k(k-1)} \sum_{i=1}^{n}\left(\hat{\theta}_{i-1}^{K}-\overline{\hat{\theta}}^{K}\right)^{2}
$$

The $K$ - repeated jackknife estimate of standard error is given by

$$
S E\left(\overline{\hat{\theta}}^{K}\right)=\sqrt{\frac{1}{k(k-1)} \sum_{i=1}^{n}\left(\hat{\theta}_{i-1}^{K}-\overline{\hat{\theta}}^{K}\right)^{2}}
$$

An approximate $(1-\alpha) \%$ confidence interval for $\theta$ is given by

$$
\overline{\hat{\theta}}^{K} \pm t_{\alpha / 2, K-1} S E\left(\overline{\hat{\theta}}^{K}\right)
$$

The mean percentage error (MPE) is

$$
\operatorname{MPE}\left(\overline{\hat{\theta}}^{K}\right)=\frac{\left[\sum_{i=1}^{n} \frac{\left|\hat{\theta}_{i-1}^{K}-\overline{\hat{\theta}}^{K}\right|}{\overline{\hat{\theta}}^{K}}\right]}{k} .
$$




\section{ADEWARA \& MBATA}

If the $K$ - repeated jackknife estimator $\overline{\hat{\theta}}^{K}$ is known from (19), then the estimate of survival function is

$$
\hat{S}^{K}(t)=\exp \left(-\frac{t_{i}-t_{0}}{\overline{\hat{\theta}}^{K}}\right) .
$$

The general iterative scheme is as follows: from a random sample $T=\left(t_{1}, t_{2}, \ldots, t_{n}\right)$

1. $\hat{\theta}_{(-1)}^{1}=\frac{1}{n-1} \sum_{i=1}^{n-1} T_{-i}$

2. $\hat{\theta}_{(-1)}^{2}=\frac{1}{n-1} \sum_{i=1}^{n-1} \hat{\theta}_{-1}^{1}$

3. $\hat{\theta}_{(-1)}^{3}=\frac{1}{n-1} \sum_{i=1}^{n-1} \hat{\theta}_{-1}^{2}$

$K . \hat{\theta}_{(-1)}^{K}=\frac{1}{n-1} \sum_{i=1}^{n-1} \hat{\theta}_{-1}^{K-1}$

Thus,

$$
\overline{\hat{\theta}}^{K}=\frac{1}{n} \sum_{i=1}^{n} \hat{\theta}_{i-1}^{K}
$$

where $K=n$ (sample size) indicates the stopping rule. Other estimators such as variance, standard error and confidence interval can be estimated as in (20), (21), (22) and (23).

This study described three types of parameter estimation methods based on re-sampling technique: the bootstrap method, the jackknife method and the $k$ repeated jackknife method. However, the intention of this study is to use Monte Carlo simulated data to compare the three methods based on mean squared error (MSE) and mean percentage error (MPE), hence survival estimation. 


\section{SURVIVAL ESTIMATION USING THREE METHODS}

\section{Data and Analysis}

Exploratory data analysis approach using simulated data generated by $\mathrm{R}$-statistical program is adopted in this research work. This is to validate the statistical assumptions of an exponential distribution. In statistics, every statistical model has its own assumptions that have to be verified and met, to provide valid results. In the case of exponential distribution, the confidence interval for the mean life of an event requires two major assumptions: the time-to-occurrence of events of interest are independent, and the time for occurrence of event is exponentially distributed. These two statistical assumptions must be satisfied for the corresponding confidence interval to cover the true mean with the prescribed probability. The simulated data is based on random generation of values which satisfies both the assumption of independence and exponentially identical distribution. Some properties of the exponential distribution are as follows: the theoretical mean and standard deviation are equal. Hence, (1) the sample values of mean and standard deviation should be close. (2) Histogram should show that the distribution is right skewed (Median < Mean). (3) A plot of Cumulative-Failure vs. Cumulative-Time should be close to linear. (4) The regression slope of Cum-Failure vs. Cum-Time is close to the failure rate. (5) A plot of Cum-Rate vs. Cum-Failure should decrease/stabilize at the failure rate level. (6) Plots of the Exponential probability and its scores should also be close to linear. Some of these properties are explained by the exploratory data analysis displayed in Figures $1, \mathrm{i}-$ xii. 
Table 1. Generation Parameters

Sample 1 (Sample size $=10, \lambda=0.5$ )

Sample 2 (Sample size $=10, \lambda=1.0$ )

Sample 3 (Sample size $=10, \lambda=1.5$ )

Sample 4 (Sample size $=10, \lambda=2.0$ )

Sample 5 (Sample size $=20, \lambda=0.5$ )

Sample 6 (Sample size $=20, \lambda=1.0$ )
Sample 7 (Sample size $=20, \lambda=1.5$ )

Sample 8 (Sample size $=20, \lambda=2.0$ )

Sample 9 (Sample size $=30, \lambda=0.5$ )

Sample 10 (Sample size $=30, \lambda=1.0$ )

Sample 11 (Sample size $=30, \lambda=1.5$ )

Sample 12 (Sample size $=30, \lambda=2.0$ )

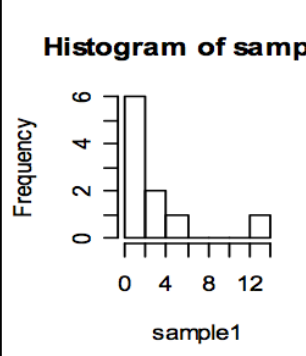

Histogram of sample

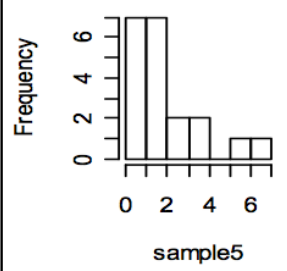

Histogram of sample

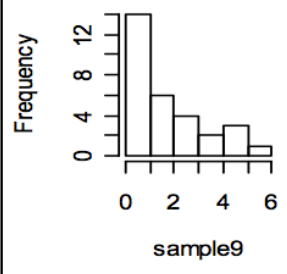

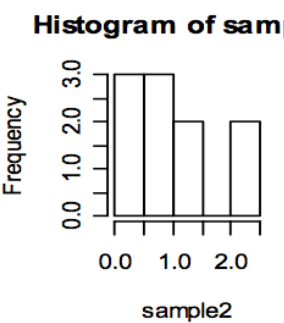

Histogram of sample

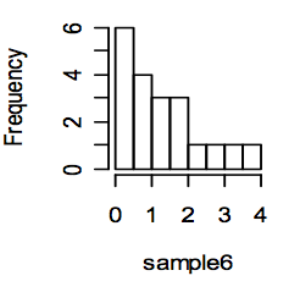

Histogram of sample 1

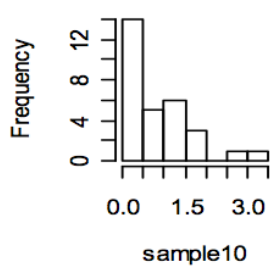

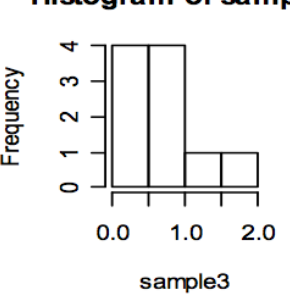

Histogram of sample

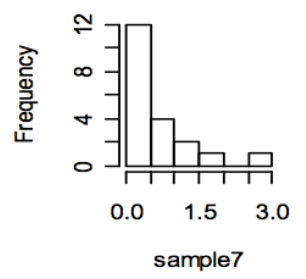

Histogram of sample

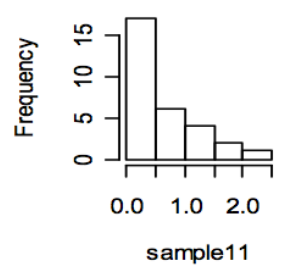

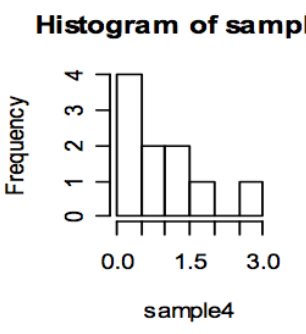

Histogram of sample

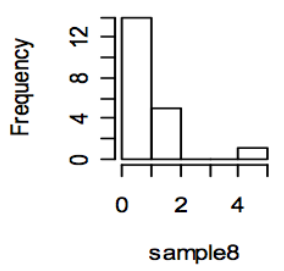

Histogram of sample

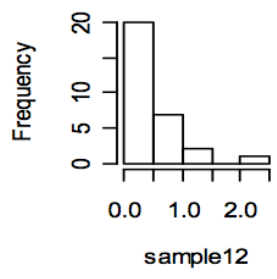

Figure 1. Histogram for each of the Randomly Generated Sample (i - xii) 


\section{SURVIVAL ESTIMATION USING THREE METHODS}

Table 2. Descriptive Statistics of samples 1 to 12

\begin{tabular}{cccccc} 
Sample (i) & $\mathbf{N}$ & $\boldsymbol{\lambda}$ & Mean & Median & Std.Dev. \\
\hline Sample 1 & 10 & 0.5 & 2.6006960 & 1.4973290 & 3.7961510 \\
Sample 2 & 10 & 1.0 & 0.9211933 & 0.7875227 & 0.7792381 \\
Sample 3 & 10 & 1.5 & 0.7320056 & 0.5990053 & 0.5961606 \\
Sample 4 & 10 & 2.0 & 0.8510537 & 0.7067248 & 0.8785794 \\
Sample 5 & 20 & 0.5 & 1.8608660 & 1.1843510 & 1.8059580 \\
Sample 6 & 20 & 1.0 & 1.2379810 & 0.9922479 & 1.0967930 \\
Sample 7 & 20 & 1.5 & 0.5363318 & 0.2470020 & 0.6540290 \\
Sample 8 & 20 & 2.0 & 0.7281072 & 0.2824484 & 1.0112260 \\
Sample 9 & 30 & 0.5 & 1.5960620 & 1.0194960 & 1.6278560 \\
Sample 10 & 30 & 1.0 & 0.8559385 & 0.5422463 & 0.8296031 \\
Sample 11 & 30 & 1.5 & 0.6353941 & 0.3864003 & 0.5880883 \\
Sample 12 & 30 & 2.0 & 0.4639596 & 0.2700518 & 0.5150784 \\
\hline
\end{tabular}

\section{Results}

The results of descriptive statistics show that as the sample sizes 10, 20 and 30 increase the mean and standard deviation are decreasing which satisfied one of the properties of that the theoretical mean and standard deviation are equal. The sample mean and standard deviation obtained are very close also as the value of $\lambda$ increases the median values obtained get smaller. Figure 1 above shows that the observed distribution agrees with the exponential distribution property 1 and property 2 described in the data above. Figures 1, i-xii show right-skewness, which supported the attribute of an exponential distribution.

Table 3 shows the parameter estimation of the three methods. The results reveal that the estimation of the bootstrap approach is better than the other two methods that is the jackknifing and $K$ repeated jackknifing. Table 4 is the result of the mean square error (MSE) of the analysis which is about the variance of the three methods. Results reveal that, as $\lambda$ values increase, the results of jackknifing and $K$ repeated jackknifing are better than the bootstrapped approach. Table 5 is the computation of the mean percentage error (MPE) the result shows that estimation of the bootstrap approach is better than the other two methods. 


\section{ADEWARA \& MBATA}

Table 3. Estimation Using the Three Methods Bootstrap, Jackknifing and $\mathrm{K}$ repeated jackknifing

\begin{tabular}{ccccc} 
& $\lambda$ & $\hat{S}_{B}(t)$ & $\hat{S}_{\text {jack }}(t)$ & $\hat{S}^{K}(t)$ \\
\hline \multirow{3}{*}{10} & 0.5 & 0.568858094 & 0.568879887 & 0.568879887 \\
& 1 & 0.476453626 & 0.476456925 & 0.476456925 \\
& 1.5 & 0.461343936 & 0.461328523 & 0.461328523 \\
& 2.0 & 0.529933691 & 0.529937819 & 0.529937819 \\
\multirow{2}{*}{20} & & & & \\
& 0.5 & 0.491722891 & 0.491777729 & 0.491777729 \\
& 1 & 0.490229963 & 0.490240047 & 0.490240047 \\
& 1.5 & 0.544947075 & 0.544930402 & 0.544930402 \\
& 2.0 & 0.553586925 & 0.553580134 & 0.553580134 \\
& & & & \\
& 0.5 & 0.527441921 & 0.527445588 & 0.527445588 \\
& 1 & 0.491819455 & 0.491882638 & 0.491882638 \\
& 1.5 & 0.491085203 & 0.491099760 & 0.491099760 \\
& 2.0 & 0.528125037 & 0.528118624 & 0.528118624 \\
\hline
\end{tabular}

Table 4. Estimation to the Bootstrap, Jackknifing and $\mathrm{K}$ repeated jackknifing using MSE methods

\begin{tabular}{|c|c|c|c|c|}
\hline & $\lambda$ & $\hat{S}_{B}(t)$ & $\hat{S}_{\text {jack }}(t)$ & $\hat{S}^{K}(t)$ \\
\hline \multirow{4}{*}{10} & 0.5 & 0.004741437 & 0.004744439 & 0.004744439 \\
\hline & 1 & 0.000554432 & 0.000554276 & 0.000554276 \\
\hline & 1.5 & 0.001494291 & 0.001495483 & 0.001495483 \\
\hline & 2.0 & 0.000896026 & 0.000896273 & 0.000896273 \\
\hline \multirow{4}{*}{20} & 0.5 & 0.000068511 & 0.000067606 & 0.000067606 \\
\hline & 1 & 0.000095454 & 0.000095257 & 0.000095257 \\
\hline & 1.5 & 0.002020240 & 0.002018741 & 0.002018741 \\
\hline & 2.0 & 0.002871559 & 0.002870831 & 0.002870831 \\
\hline \multirow{4}{*}{30} & 0.5 & 0.000753059 & 0.000753260 & 0.000753260 \\
\hline & 1 & 0.000066921 & 0.000065892 & 0.000065892 \\
\hline & 1.5 & 0.000079474 & 0.000079214 & 0.000079214 \\
\hline & 2.0 & 0.000791018 & 0.000790657 & 0.000790657 \\
\hline
\end{tabular}




\section{SURVIVAL ESTIMATION USING THREE METHODS}

Table 5. Bootstrap, Jackknifing and K-repeated jackknifing using MPE methods

\begin{tabular}{ccccc} 
& $\lambda$ & $\hat{S}_{B}(t)$ & $\hat{S}_{\text {jack }}(t)$ & $\hat{S}^{K}(t)$ \\
\hline \multirow{3}{*}{10} & 0.5 & 0.137716188 & 0.137759774 & 0.137759774 \\
& 1 & 0.047092748 & 0.047086150 & 0.047086150 \\
& 1.5 & 0.077312128 & 0.077342954 & 0.077342954 \\
& 2.0 & 0.059867382 & 0.059875638 & 0.059875638 \\
20 & & & & \\
& 0.5 & 0.016554218 & 0.016444542 & 0.016444542 \\
& 1 & 0.019540074 & 0.019519906 & 0.019519906 \\
& 1.5 & 0.089894150 & 0.089860804 & 0.089860804 \\
& 2.0 & 0.107173850 & 0.107160268 & 0.107160268 \\
& & & & \\
& 0.5 & 0.054883842 & 0.054891176 & 0.054891176 \\
& 1 & 0.016361090 & 0.016234724 & 0.016234724 \\
& 1.5 & 0.017829594 & 0.017800480 & 0.017800480 \\
& 2.0 & 0.056250074 & 0.056237248 & 0.056237248 \\
\hline
\end{tabular}

Table 6. Survival Estimation Using the Three Methods with Respect to MSE and MPE

\section{BOOTSTRAP METHOD (1)}

\begin{tabular}{cccccc} 
Size & $\lambda$ & $\hat{S}_{B}(t)$ & MSE & MPE & REMARK \\
\hline \multirow{3}{*}{10} & 0.5 & 0.568858094 & 0.004741437 & 0.137716188 & 1 \\
& 1 & 0.476453626 & 0.000554432 & 0.047092748 & 2,3 \\
& 1.5 & 0.461343936 & 0.001494291 & 0.077312128 & 1 \\
& 2.0 & 0.529933691 & 0.000896026 & 0.059867382 & 1 \\
& & & & & \\
20 & 0.5 & 0.491722891 & 0.000068511 & 0.016554218 & 2,3 \\
& 1 & 0.490229963 & 0.000095454 & 0.019540074 & 2,3 \\
& 1.5 & 0.544947075 & 0.002020240 & 0.089894150 & 2,3 \\
& 2.0 & 0.553586925 & 0.002871559 & 0.107173850 & 2,3 \\
& & & & & \\
30 & 0.5 & 0.527441921 & 0.000753059 & 0.054883842 & 1 \\
& 1 & 0.491819455 & 0.000066921 & 0.016361090 & 2,3 \\
& 1.5 & 0.491085203 & 0.000079474 & 0.017829594 & 2,3 \\
& 2.0 & 0.528125037 & 0.000791018 & 0.056250074 & 2,3 \\
\hline
\end{tabular}


ADEWARA \& MBATA

Table 6, cont'd. Survival Estimation Using the Three Methods with Respect to MSE and MPE

\begin{tabular}{|c|c|c|c|c|c|}
\hline \multirow[b]{2}{*}{ Size } & \multicolumn{5}{|c|}{ JACKKNIFE METHOD (2) } \\
\hline & $\lambda$ & $\hat{S}_{\text {jack }}(t)$ & MSE & MPE & REMARK \\
\hline \multirow{4}{*}{10} & 0.5 & 0.568858094 & 0.004741437 & 0.137716188 & 1 \\
\hline & 1 & 0.476453626 & 0.000554432 & 0.047092748 & 2,3 \\
\hline & 1.5 & 0.461343936 & 0.001494291 & 0.077312128 & 1 \\
\hline & 2.0 & 0.529933691 & 0.000896026 & 0.059867382 & 1 \\
\hline \multirow{4}{*}{20} & 0.5 & 0.491722891 & 0.000068511 & 0.016554218 & 2,3 \\
\hline & 1 & 0.490229963 & 0.000095454 & 0.019540074 & 2,3 \\
\hline & 1.5 & 0.544947075 & 0.002020240 & 0.089894150 & 2,3 \\
\hline & 2.0 & 0.553586925 & 0.002871559 & 0.107173850 & 2,3 \\
\hline \multirow{4}{*}{30} & 0.5 & 0.527441921 & 0.000753059 & 0.054883842 & 1 \\
\hline & 1 & 0.491819455 & 0.000066921 & 0.016361090 & 2,3 \\
\hline & 1.5 & 0.491085203 & 0.000079474 & 0.017829594 & 2,3 \\
\hline & 2.0 & 0.528125037 & 0.000791018 & 0.056250074 & 2,3 \\
\hline
\end{tabular}

K-REPEATED JACKKNIFE METHOD (3)

\begin{tabular}{rrrrrc} 
Size & $\lambda$ & $\hat{S}^{K}(t)$ & MSE & MPE & REMARK \\
\hline \multirow{3}{*}{10} & 0.5 & 0.568858094 & 0.004741437 & 0.137716188 & 1 \\
& 1 & 0.476453626 & 0.000554432 & 0.047092748 & 2,3 \\
& 1.5 & 0.461343936 & 0.001494291 & 0.077312128 & 1 \\
& 2.0 & 0.529933691 & 0.000896026 & 0.059867382 & 1 \\
& & & & & \\
20 & 0.5 & 0.491722891 & 0.000068511 & 0.016554218 & 2,3 \\
& 1 & 0.490229963 & 0.000095454 & 0.019540074 & 2,3 \\
& 1.5 & 0.544947075 & 0.002020240 & 0.089894150 & 2,3 \\
& 2.0 & 0.553586925 & 0.002871559 & 0.107173850 & 2,3 \\
& & & & & \\
& 0.5 & 0.527441921 & 0.000753059 & 0.054883842 & 1 \\
& 1 & 0.491819455 & 0.000066921 & 0.016361090 & 2,3 \\
& 1.5 & 0.491085203 & 0.000079474 & 0.017829594 & 2,3 \\
& 2.0 & 0.528125037 & 0.000791018 & 0.056250074 & 2,3 \\
\hline
\end{tabular}




\section{SURVIVAL ESTIMATION USING THREE METHODS}

\section{Discussion}

The comparison of parametric estimators of exponential distribution using Bootstrap, Jackknife, and $K$-Repeated Jackknife methods indicates that the estimates of the population parameter are very close which implies that the estimators are unbiased. A comparison of the mean square error (MSE) and mean percentage error (MPE) of the estimators shows that $K$-Repeated Jackknife method has a minimum variance unbiased estimator (MVUE); irrespective of the sample size whether it is small or large at any given values of lambda $(\lambda)$. The three methods are used to estimate the survival function for exponential distribution and its mean square error (MSE) and mean percentage error (MPE). The results can be deduced that the performance of the two jackknife procedures over the bootstrap procedure is $66.67 \%$ to $33.33 \%$. This result has been able to show the effect or influence of jackknife method, especially the $k$-repeated procedure on error reduction in estimating population parameter.

\section{Conclusion}

This study demonstrates that both methods of re-sampling technique are very efficient in estimating the population parameters and their mean square errors (MSE), as viewed by Efron (1998). These methods were used to find the best minimum variance unbiased estimator, using mean square error (MSE) and mean percentage error (MPE). The estimates of the two-parameter exponential distribution are used to estimate the survival probability. The attractiveness of jackknifing and bootstrapping is that they provide investigators with an important and unattainable type of information. Jackknifing and bootstrapping have their limitations and inherent assumptions as all statistical procedures do. The three methods are computationally intensive. However, these techniques represent an important step in refining the process of data analysis more especially the $k$ repeated procedure. Hence, it can be deduced that bootstrapping is a method for evaluating the variance of an estimator while jackknife is a method for reducing the bias of an estimator, and evaluating the variance of an estimator. This is clearly shown in the MSE results. The MSE value is reduced using the $K$-repeated jackknife method. 
ADEWARA \& MBATA

\section{References}

Bradley, J. U. (1968). Distribution-free statistical tests. Englewood Cliff, NJ: Prentice-Hall.

Collett, D. (1994). Modeling survival data in medical research. London: Chapman and Hall.

Conover, W. J. (1980). Practical nonparametric statistics. New York: John Wiley and Sons.

Cox, D. R., \& Oakes, D. (1984). Analysis of survival data. New York: Chapman and Hall.

Crowley, P. (1992). Resampling methods for computation-intensive data analysis in ecology and evolution. Annual Review of Ecological Systems, 23, 405447.

Efron, B., \& Gong, G. (1983). A leisurely look at the bootstrap, the jackknife, and cross-validation. The American Statistician, 37, 36-48.

Efron, B., \& Tibshirani, R. (1998). An introduction to the bootstrap. Boca Raton, FL: Chapman \& Hall/CRC.

Fernández, A. J. (2000). Estimation and Hypothesis Testing for Exponential Lifetime Models with Double Censoring and Prior Information. Journal of Economic and Social Research, 2(2), 1-17.

Good, P. L. (2005). Resampling methods (3rd ed.). Berlin: Birkhauser.

Lawless, J. F. (1982). Statistical models and methods for lifetime data. New York: John Wiley and Sons.

Miller, R. G. (1974a). An unbalanced jackknife. Annals of Statistics, 2, 880891.

Miller, R. G. (1974b). The jackknife-a review. Biometrika, 61, 1-15.

Quenouille, M. H. (1949). Approximate tests of correlation in time-series. Journal of the Royal Statistical Society, Series B, 11, 68-84.

Quenouille, M. H. (1956). Notes on bias in estimation. Biometrika, 43, 353360.

Rizzo, M. L. (2008). Statistical computing with R. Boca Raton, FL: Chapman and Hall/CRC.

Suzuki, K. (1985a). Nonparametric estimation of lifetime distributions from a record of failures and follow-ups. Journal of the American Statistical Association, 80, 68-72. 


\section{SURVIVAL ESTIMATION USING THREE METHODS}

Suzuki, K. (1985b). Estimation of lifetime parameters from incomplete field

data. Technometrics, 27, 263-271. 
ADEWARA \& MBATA

\section{Appendix}

\section{Simulated Data}

Table A1. Sample Size $=10$

\begin{tabular}{ccccc}
$\mathbf{S} / \mathbf{N}$ & Sample $\mathbf{1}(\boldsymbol{\lambda}=\mathbf{0 . 5})$ & Sample $\mathbf{2}(\boldsymbol{\lambda}=\mathbf{1 . 0})$ & Sample $\mathbf{3}(\boldsymbol{\lambda}=\mathbf{1 . 5})$ & Sample $\mathbf{4}(\boldsymbol{\lambda}=\mathbf{2 . 0})$ \\
\hline 1 & 0.38672500 & 2.27994293 & 0.20192160 & 0.01058359 \\
2 & 0.11665480 & 0.71411938 & 0.48301090 & 2.51951031 \\
3 & 0.12661390 & 0.75298901 & 0.12473380 & 1.94495769 \\
4 & 4.42286640 & 2.19289233 & 1.99662440 & 0.67531853 \\
5 & 2.00704600 & 0.04442099 & 0.67780240 & 1.05647689 \\
6 & 2.34689070 & 0.35021824 & 0.75981590 & 0.01373747 \\
7 & 0.86211020 & 1.01674933 & 0.87373650 & 0.08979746 \\
8 & 1.03118540 & 0.82205630 & 0.21055390 & 0.73813115 \\
9 & 12.74339800 & 0.02251542 & 1.47164790 & 0.07333057 \\
10 & 1.96347260 & 1.01602885 & 0.52020820 & 1.38869338 \\
\hline
\end{tabular}

Table A2. Sample Size $=20$

\begin{tabular}{ccccc}
$\mathbf{S} / \mathbf{N}$ & Sample $\mathbf{5}(\boldsymbol{\lambda}=\mathbf{0 . 5})$ & Sample $\mathbf{6}(\boldsymbol{\lambda}=\mathbf{1 . 0})$ & Sample $\mathbf{7}(\boldsymbol{\lambda}=\mathbf{1 . 5})$ & Sample $8(\boldsymbol{\lambda}=\mathbf{2 . 0})$ \\
\hline 1 & 2.62129072 & 0.13858938 & 0.61025422 & 0.20816883 \\
2 & 3.66009415 & 0.23677409 & 1.39562321 & 0.10508682 \\
3 & 0.28671927 & 0.65882621 & 1.64585069 & 0.16343073 \\
4 & 0.53614812 & 2.03913598 & 1.11779841 & 4.47429656 \\
5 & 1.67262059 & 3.07310425 & 0.64918675 & 0.31910786 \\
6 & 0.08521250 & 0.85047816 & 0.05778008 & 0.21707275 \\
7 & 0.84341716 & 0.93587816 & 0.04898416 & 0.04360368 \\
8 & 1.87988871 & 3.97283307 & 0.12098188 & 0.11505109 \\
9 & 3.13213741 & 1.91406287 & 0.55509004 & 0.24578897 \\
10 & 6.82508233 & 1.04861761 & 0.19238185 & 1.05875641 \\
11 & 0.02900937 & 0.03350444 & 0.26953211 & 0.00355591 \\
12 & 1.74900498 & 1.30175357 & 0.01133644 & 0.05922794 \\
13 & 1.24431388 & 1.52538027 & 0.28231025 & 0.20815442 \\
14 & 1.12438751 & 0.06504098 & 2.50193518 & 1.48214568 \\
15 & 1.02901637 & 1.90192968 & 0.52524965 & 0.89694421 \\
16 & 2.19818977 & 0.71117769 & 0.20890800 & 1.53327300 \\
17 & 5.75705560 & 2.66119925 & 0.12742349 & 1.11055824 \\
18 & 0.67605113 & 1.38839741 & 0.09933566 & 0.81368075 \\
19 & 0.79778248 & 0.30239189 & 0.08220231 & 0.46919296 \\
20 & 1.06989471 & 0.00054309 & 0.22447181 & 1.03504680 \\
\hline
\end{tabular}




\section{SURVIVAL ESTIMATION USING THREE METHODS}

Table A3. Sample Size $=30$

\begin{tabular}{ccccc}
$\mathbf{S} / \mathbf{N}$ & Sample $\mathbf{9}(\boldsymbol{\lambda}=\mathbf{0 . 5})$ & Sample $\mathbf{1 0}(\boldsymbol{\lambda}=\mathbf{1 . 0})$ & Sample $\mathbf{1 1}(\boldsymbol{\lambda}=\mathbf{1 . 5})$ & Sample $\mathbf{1 2}(\boldsymbol{\lambda}=\mathbf{2 . 0})$ \\
\hline 1 & 1.09871091 & 0.17085314 & 0.24591320 & 0.03006484 \\
2 & 1.30597223 & 0.31266839 & 0.78554402 & 0.01316790 \\
3 & 0.43742079 & 0.21960578 & 0.26193004 & 0.08580695 \\
4 & 0.57034193 & 1.09603105 & 0.07651001 & 0.06684230 \\
5 & 1.02475744 & 0.54796524 & 0.29620540 & 0.75349995 \\
6 & 1.53172531 & 0.13561858 & 0.58560088 & 0.56383185 \\
7 & 0.57173448 & 0.46805576 & 0.56401687 & 0.10014385 \\
8 & 3.18835121 & 0.58675535 & 0.41054238 & 0.39468972 \\
9 & 1.01423546 & 0.41116558 & 1.42498655 & 0.26076246 \\
10 & 0.05104055 & 3.42353290 & 0.03212381 & 0.38563026 \\
11 & 2.99399940 & 1.09817464 & 0.19768464 & 0.96558979 \\
12 & 2.95112802 & 0.35962685 & 0.24295821 & 0.27934118 \\
13 & 4.74244122 & 1.98862082 & 0.16056365 & 0.01547041 \\
14 & 0.04628853 & 1.11964839 & 0.24140637 & 0.34343548 \\
15 & 5.35809191 & 0.48539163 & 0.06956623 & 0.80092480 \\
16 & 4.26185504 & 1.09365222 & 1.49188159 & 1.49780414 \\
17 & 0.04367701 & 0.73949713 & 0.22345808 & 0.90349970 \\
18 & 0.05851474 & 0.34345758 & 1.56937093 & 0.18701462 \\
19 & 0.46455153 & 0.29243694 & 0.59195204 & 0.00288255 \\
20 & 0.09371455 & 0.25904558 & 0.42176436 & 0.74330912 \\
21 & 2.96970220 & 1.79180600 & 1.11983745 & 0.25455636 \\
22 & 0.54133977 & 0.01809066 & 0.28416911 & 0.81341579 \\
23 & 1.29204462 & 2.94010580 & 0.65638599 & 0.10230827 \\
24 & 0.02923826 & 0.79327459 & 0.12515747 & 2.24807427 \\
25 & 2.28724168 & 1.13577062 & 2.26479793 & 0.08718112 \\
26 & 3.62406597 & 0.05051664 & 1.73361763 & 1.15865739 \\
27 & 0.55831489 & 0.53652729 & 0.36225826 & 0.02484439 \\
28 & 4.24494133 & 1.08361205 & 1.41035274 & 0.48756216 \\
29 & 0.13192788 & 0.25488727 & 0.93471466 & 0.25596331 \\
30 & 0.39449119 & 1.92176066 & 0.27655395 & 0.09251206 \\
\hline & & & &
\end{tabular}

